\title{
Thermal recalcitrance of the organic D-rich component of ordinary
}

\section{chondrites}

\author{
L. Remusat ${ }^{1 *}$, L. Piani $^{1,2}$ and S. Bernard ${ }^{1}$
}

${ }^{1}$ Institut de Minéralogie, de Physique des Matériaux, et de Cosmochimie (IMPMC), UMR

CNRS 7590 - Sorbonne Universités - UPMC - IRD - Muséum National d'Histoire Naturelle,

57 rue Cuvier, Case 52, 75231 Paris Cedex 5, France.

${ }^{2}$ Department of Natural History Sciences, Hokkaido University, Sapporo 060-0810, Japan.

\section{*Corresponding author: remusat@mnhn.fr}

\begin{abstract}
Carbonaceous and ordinary chondrites (CCs and OCs) contain insoluble organic matter (IOM) with large D-excess compared to other objects in the solar system. The higher the temperature experienced by CCs, the lower the D/H ratio of their IOM. It seems to be the opposite for OCs. Here, we report NanoSIMS H- (and N-) isotopic imaging of IOM of three OCs that experienced thermal metamorphism in the sequence Semarkona, Bishunpur and GRO 95502. In addition, we performed flash heating experiments on the IOM of GRO 95502 at $600^{\circ} \mathrm{C}$ and characterized the residues using NanoSIMS, Raman and XANES spectroscopy. The present study shows that, in contrast to IOM of CI, CM and CR, IOM of OCs exhibits very few D-rich (or ${ }^{15} \mathrm{~N}$-rich) hotspots. Furthermore, although the evolution of the molecular structure of OC and CC IOM is similar upon heating, their D/H ratios do not follow the same trend: the $\mathrm{D} / \mathrm{H}$ of $\mathrm{OC}$ IOM drastically increases while the $\mathrm{D} / \mathrm{H}$ of $\mathrm{CC}$ IOM decreases. In contrast to CC IOM, the D-rich component of which does not survive at high temperatures, the present results highlight the thermal recalcitrance of the D-rich component of OC IOM.
\end{abstract}

(1)

3


26 This suggests that CCs and OCs did not accrete the same organic material, thereby 27 challenging the hypothesis of a common precursor on chondritic parent bodies. The present 28 results support the hypothesis that OC IOM contains an organic component that could 29 originate from the interstellar medium.

30

31 Keywords: insoluble organic matter; chondrite; parent body processes; NanoSIMS; hydrogen

32 and nitrogen isotopes; solar system 
Chondrites are often considered to be the witnesses of solar system formation

(Weisberg et al., 2006). Their components are believed to have formed during the collapse of

the parent molecular cloud and solar nebula evolution. They are consequently the targets for many studies aiming to understand the processes of formation of the constituents of planetesimals and planets. The origin of volatile reservoirs in the early solar system is the subject of intense debate, with implications for the origin of water and life on Earth. In this respect, carbonaceous chondrites (CCs) have received most of the attention because they are the most organic- and water-rich chondrites (CCs may contain up to a few weight percent of carbon). Two types of organic matter are usually distinguished (Remusat, 2015): the organic matter that is soluble in water and organic solvents, and the insoluble organic matter (IOM). The IOM constitutes the major part of the total organic material and is generally studied after isolation from the minerals by acid treatments using $\mathrm{HF}-\mathrm{HCl}$ (Robert and Epstein, 1982) or CsF-HCl, (Alexander et al., 2007).

The molecular, structural and chemical composition of CC IOM has been extensively documented (Remusat, 2015 and references herein). It corresponds to a macromolecule made of small aromatic units linked by short aliphatic chains that contains hetero-elements $(\mathrm{N}, \mathrm{S}$, O) (Hayatsu et al., 1980; Remusat et al., 2005; Cody and Alexander, 2005; Orthous-Daunay et al., 2010) and organic radicals (Binet et al., 2002; Binet et al., 2004). Type 1 and 2 CC IOM is enriched in heavy isotopes of $\mathrm{H}$ and $\mathrm{N}$ relative to terrestrial organic matter, with the exception of some CMs having near-atmospheric IOM N isotopic compositions (Robert and Epstein, 1982; Yang and Epstein, 1983; Alexander et al., 2007; Alexander et al., 2010), and exhibits micron-sized hotspots enriched in $\mathrm{D}$ and ${ }^{15} \mathrm{~N}$ (Busemann et al., 2006; Nakamura- 
remnants of interstellar organic compounds (Busemann et al., 2006; Nakamura-Messenger et al., 2006) or evidence of an early irradiation of the solar system (Remusat et al., 2009). Of note, at least for Murchison (CM chondrite), these hotspots disappear when the IOM is submitted to thermal treatment at $600^{\circ} \mathrm{C}$ under He atmosphere (Remusat et al., 2009).

Despite the abundance of available samples, much less is known about organic matter in ordinary chondrites (OCs). In contrast to most $\mathrm{CCs}$, which experienced aqueous alteration on parent bodies, most OCs experienced thermal metamorphism that may have led to the complete degradation of organics (Yang and Epstein, 1983; Alexander et al., 2010). Although the two falls Semarkona (LL3.0) and Bishunpur (LL3.15) exhibit some signs of secondary hydrous alteration (i.e. processes on the parent body, Alexander et al., 1989; Sears et al., 1995). Raman spectroscopy investigations on IOM have shown that Semarkona is, by far, the most pristine unequilibrated ordinary chondrite (UOC). IOM experienced a metamorphic temperature peak well below $250^{\circ} \mathrm{C}$ (Quirico et al., 2003; Busemann et al., 2007); this temperature is consistent with estimates from mineral assemblage $\left(\mathrm{T}<260^{\circ} \mathrm{C}\right.$ - Alexander et al., 1989) and sulfide thermometry $\left(230^{\circ} \mathrm{C}\right.$ - Zanda et al. 1995). The IOM of OCs is generally richer in $\mathrm{D}$ than that of $\mathrm{CCs}$ and exhibits anticorrelated $\mathrm{D} / \mathrm{H}$ and $\mathrm{H} / \mathrm{C}$ ratios, in contrast to $\mathrm{CC}$ IOM (Alexander et al., 2007, 2010). No D- or ${ }^{15} \mathrm{~N}$-rich hotspot has ever been reported in OC IOM, as recently illustrated by NanoSIMS images of the IOM of the Krymka meteorite (LL3.2) showing no heterogeneities in D/H (Alexander et al., 2010).

The present study reports NanoSIMS H- and N- isotopic imaging of IOM of three UOCs that experienced different thermal metamorphism: Semarkona (LL3.0), Bishunpur (LL3.15) and Grosvenor Mountains (GRO) 95502 (L3.2). In addition, we document the molecular signature and structural properties of the IOM of GRO 95502 before and after flash heating experiments. The results highlight the thermal recalcitrance of the D-rich component 
83 of OC IOM and suggest that the original precursors of $\mathrm{CC}$ and $\mathrm{OC}$ organic matter were 84 different. 
2.1 Samples

IOM has been isolated from three UOCs of increasing metamorphic grades:

Semarkona (LL3.0), Bishunpur (LL3.15), and GRO 95502 (L3.2). Semarkona and Bishunpur IOM was prepared by Yang and Epstein (1983) by classical $\mathrm{HF} / \mathrm{HCl}$ dissolution. This IOM has been stored since then at room temperature, in a glass vial sealed by a Teflon cap and protected from the sunlight. Fifty milligrams of GRO 95502 IOM (hereafter called GRO IOM) were recently isolated from $20 \mathrm{~g}$ of meteorite using a similar protocol (Piani et al., 2012a). An aliquot of GRO IOM was subjected to flash pyrolysis at $600^{\circ} \mathrm{C}$ under a $\mathrm{He}$ atmosphere. The (organic) residue was recovered and is hereafter called GROpyr. Orgueil IOM, prepared by Remusat et al. (2005), was used as a reference material.

Aliquots of these IOM samples were pressed into cleaned indium foil and gold coated (20 nm thick) to improve charge compensation during NanoSIMS imaging. Portions of GRO IOM and GROpyr samples were also finely powdered in an agate mortar and deposited on $\mathrm{Si}_{3} \mathrm{~N}_{4}$ windows for Raman and XANES (X-ray absorption near edge structure) investigations.

102

\subsection{NanoSIMS settings}

Isotopic images were acquired using the Cameca NanoSIMS 50 installed at the National Museum of Natural History in Paris, France. A $16 \mathrm{keV}$ primary $\mathrm{Cs}^{+}$beam was used to collect the secondary ions $\mathrm{H}^{-}$and $\mathrm{D}^{-}$during a first run (to obtain $\delta \mathrm{D}$ images), and ${ }^{16} \mathrm{O}^{-},{ }^{12} \mathrm{C}_{2}^{-}$

$107,{ }^{12} \mathrm{C}^{14} \mathrm{~N}^{-},{ }^{12} \mathrm{C}^{15} \mathrm{~N}^{-}$and ${ }^{32} \mathrm{~S}^{-}$during a second run (for $\mathrm{N} / \mathrm{C}$ and $\delta^{15} \mathrm{~N}$ images). The use of the ion 108 ratio ${ }^{12} \mathrm{C}^{14} \mathrm{~N}^{-} /{ }^{12} \mathrm{C}_{2}^{-}$reduces topographic effects on the $\mathrm{N} / \mathrm{C}$ measurements (Thomen et al., 109 2014; Alleon et al., 2015). The primary beam was set to $8 \mathrm{pA}$ for $\mathrm{H}$ isotope and to $3 \mathrm{pA}$ for $\mathrm{N}$ 110 isotope measurements, leading to spatial resolutions of about 300 and $150 \mathrm{~nm}$, respectively. 
111 We collected $256 \times 256$ pixel images covering $20 \times 20 \mu \mathrm{m}^{2}$ with a raster speed of $2 \mathrm{~ms} /$ pix. Prior

112 to each analysis, a $25 \times 25 \mu \mathrm{m}^{2}$ presputtering was applied using a $600 \mathrm{pA}$ primary current for 6

113 minutes (corresponding to a $\mathrm{Cs}^{+}$fluence of $2.2 \times 10^{17}$ at. $\mathrm{cm}^{-2}$ ) in order to remove the gold coat,

114 clean the surface, and reach the sputtering steady state (Thomen et al., 2014). We used

115 Hamamatsu discrete dynode electron multipliers with a dead time of $44 \mathrm{~ns}$ in multicollection

116 mode. For $\mathrm{H}$ isotopes, the mass spectrometer was set to a mass resolving power of 4000, and

117 to 8000 for $\mathrm{N}$ isotopes to resolve isobaric interferences, such as ${ }^{12} \mathrm{C}^{14} \mathrm{~N}^{-}$from ${ }^{12} \mathrm{C}_{2} \mathrm{H}_{2}^{-}$and ${ }^{32} \mathrm{~S}^{-}$

118 from ${ }^{16} \mathrm{O}_{2}^{-}$. The vacuum in the analysis chamber never exceeded $5.10^{10}$ torr.

119 The collected NanoSIMS data were then processed with the L'Image software 120 developed by Larry Nittler, Carnegie Institution in Washington DC, USA. Each image being a 121 stack of several frames, the first step consists of aligning each frame using a correlation 122 algorithm and applying the same shift in $\mathrm{X}$ and $\mathrm{Y}$ to all the pixels of a single frame. Then, 123 ratio images can be generated. Each ratio is corrected using a calibration line (see 124 supplementary online material), determined by measuring four known reference samples 125 (which in the meantime allow checking the instrument stability): a terrestrial Type 3 kerogen, 126 a charcoal and the IOM of Orgueil and GRO 95502 (previously measured by gas source mass 127 spectrometry by Alexander et al., (2007). Each calibration line is calculated using a linear 128 regression and the $\mathrm{R}$ program was used to determine uncertainties associated with the 129 calibration (Table S1). All the uncertainties reported in this study are one standard deviation; 130 we propagated (using quadratic sum) counting statistics uncertainties on each regions of 131 interest (ROI) and uncertainties arising from the calibration lines, reflecting the external 132 precision of our measurements (e.g., the standard deviation for independent measurements 133 repeated several times on the same sample).

134 Isotopic ratios are expressed in delta units, following the relation: $\delta(\%)=\left(R_{\text {sple }} / R_{\text {std }}-1\right)$

$135 \times 1000$, with $R_{\text {sple }}$ being the sample isotopic ratio and $R_{\text {std }}$ a reference ratio, such as Standard 
136 Mean Ocean Water (SMOW: $\mathrm{D} / \mathrm{H}=155.76 \times 10^{-6}$ ) for $\mathrm{H}$ isotopes and air for $\mathrm{N}$ isotopes $137\left({ }^{15} \mathrm{~N} /{ }^{14} \mathrm{~N}=3.67 \times 10^{-3}\right)$. Isotopic anomalies in $\mathrm{D}\left(\right.$ or in $\left.{ }^{15} \mathrm{~N}\right)$ are defined as ROIs consisting of at

138 least 50 pixels $\left(0.3 \mu^{2}\right)$ meeting individually the requirement $\mid(\mathrm{D} / \mathrm{H})_{\text {pixel }}(\mathrm{D} / \mathrm{H})_{\text {average }} / / \sigma>2$ 139 (or $\left.\mid\left({ }^{15} \mathrm{~N} /{ }^{14} \mathrm{~N}\right)_{\text {pixel }}-\left({ }^{15} \mathrm{~N} /{ }^{14} \mathrm{~N}\right)_{\text {average }} / \sigma>2\right)$, where $(\mathrm{D} / \mathrm{H})_{\text {pixel }}\left(\right.$ or $\left.\left({ }^{15} \mathrm{~N} /{ }^{14} \mathrm{~N}\right)_{\text {pixel }}\right)$ is the isotopic ratio 140 of the pixel, $(\mathrm{D} / \mathrm{H})_{\text {average }}\left(\right.$ or $\left.\left({ }^{15} \mathrm{~N} /{ }^{14} \mathrm{~N}\right)_{\text {average }}\right)$ the average ratio of the image and $\sigma$ the statistical 141 error for each pixel (Table S2). Anomalies can be significantly positive or negative, reflecting 142 enrichment or depletion in $\mathrm{D}$ ( or ${ }^{15} \mathrm{~N}$ ) compared to the average of the image, respectively. The 143 heterogeneity in the $\mathrm{D} / \mathrm{H}$ ratio can be investigated using pixel histograms showing the 144 distributions of $\delta \mathrm{D}\left(\right.$ or $\delta^{15} \mathrm{~N}$ ) values of individual pixels within each image. The occurrence of 145 positive micron-scale anomalies can be noticed on these histograms. They result in tails on 146 the right side of histograms but do not affect the full width at half maximum (FWHM). Of 147 note, some of these anomalies may correspond to presolar grains (e.g. Floss and Stadermann, 148 2009). Data are reported in the supplementary online material (tables S2 and S3).

\subsection{Raman and STXM/XANES characterization}

Raman data were collected on powdered samples using the Renishaw INVIA microspectrometer operating at IMPMC following the procedure described in (Bernard et al., 2008). Twenty spectra were collected on each IOM to capture their homogeneous or

154 heterogeneous nature. Spectra were measured from 500 to $3500 \mathrm{~cm}^{-1}$ at constant room 155 temperature using the $514.5 \mathrm{~nm}$ wavelength of a $50 \mathrm{~mW}$ Modulaser Argon laser (green laser)

156 focused on the sample through a Leica DMLM microscope with a long working distance 50X 157 objective $(\mathrm{NA}=0.55)$. This configuration yields a planar resolution of $\sim 1 \mu \mathrm{m}$ for a laser 158 power delivered at the sample surface set at around $250 \mu \mathrm{W}$ to prevent irreversible thermal 159 damage due to laser-induced heating (Beyssac et al., 2003). A grating with 1800 lines/mm 160 disperses light and the signal was analyzed with a RENCAM CCD detector. Measurements 
161 are performed with a circularly polarized laser using a $1 / 4$ wavelength plate placed before the microscope in order to limit polarization effects.

164 microscope) located on beamline 5.3.2.2 (STXM Polymer beamline - (Kilcoyne et al., 2003))

165 at the Advanced Light Source (ALS). This beamline uses soft X-rays (250 - $600 \mathrm{eV})$

166 generated via a bending magnet while the electron current in the storage ring is held constant

167 in top-off mode at $500 \mathrm{~mA}$ at a storage ring energy of $1.9 \mathrm{GeV}$. The microscope chamber is 168 evacuated to 100 mTorr after sample insertion and back-filled with He. Energy calibration is 169 accomplished using the well-resolved 3p Rydberg peak at $294.96 \mathrm{eV}$ of gaseous $\mathrm{CO}_{2}$ for the 170 C K-edge. Alignment of images of stacks and extraction of XANES spectra were done using 171 the aXis2000 software (ver2.1n) while normalization to the carbon quantity was done using 172 the Athena software package (Ravel and Newville, 2005). The C-XANES spectra shown in 173 the present study are for homogeneous organic-rich areas of several hundreds of nanometers.

174 Although radiation damage per unit of analytical information was shown to be typically 1001751000 times lower in STXM-based XANES spectroscopy than in TEM-based EELS 176 (Hitchcock et al., 2008), the C-XANES data shown here were collected following the 177 procedures for X-ray microscopy studies of radiation sensitive samples recommended by 178 Wang et al. (2009). Extensive databases of reference XANES spectra (measured on hundreds 179 of C-containing compounds at the C K-edge, sometimes supported by theoretical calculations 180 using multiple scattering approaches) are available (Solomon et al., 2009). 


\subsection{NanoSIMS imaging of $H$ isotopes}

The spatial distributions of hydrogen isotopes in the OC IOM are remarkably different from that of the Orgueil IOM (Remusat et al., 2009). Semarkona and Bishunpur IOM exhibit different isotopic compositions depending on the measured area (Figure 1). Despite their Drich nature, the IOM of OCs exhibits fewer D anomalies than does Orgueil IOM (Busemann et al., 2006; Nakamura-Messenger et al., 2006; Remusat et al., 2009). In addition, the intensities and sizes of isotopic anomalies in OC IOM differ from those in Orgueil and other CC IOM (Figure 2 and table S2). Orgueil IOM exhibits numerous micron-scale D anomalies

193 (25 over $800 \mu^{2}$, with $2000 \%$ o $\delta \mathrm{D}<4000 \%$ ) while the average $\delta \mathrm{D}$ value is 194 indistinguishable from one region to another. In contrast, only two micron-scale D anomalies 195 are detected in Semarkona IOM (one positive with $\delta \mathrm{D}=5000 \%$ and one negative with $196 \delta \mathrm{D}=300 \%$ while entire regions may display significant $\mathrm{D}$-enrichments $(\delta \mathrm{D}=5000 \%$ for 197 instance over the area 2 in Figure 1) compared to adjacent ones ( $\delta \mathrm{D}=2000 \%$ over the area 1

198 in Figure 1). Bishunpur IOM displays large regions with $\delta \mathrm{D}$ values ranging from $800 \%$ to $1992700 \%$ and exhibits five positive (up to $\delta \mathrm{D}=4000 \%$ ) and three negative (around $\delta \mathrm{D}=200 \%$ ) 200 micron-scale anomalies over $1200 \mu^{2}$. Despite three positive $(5500 \%<<\mathrm{D}<6300 \%)$ and 201 four negative $(1200 \%$ o $<\mathrm{D}<1500 \%$ ) anomalies, the GRO IOM exhibits a quite 202 homogeneous D/H ratio (considering about $600 \mu \mathrm{m}^{2}$ analyzed in this study), consistent with 203 previous measurements (Piani et al., 2012a; Piani et al., 2015). The flash pyrolysis at $600^{\circ} \mathrm{C}$ 204 of this IOM induces a D-enrichment by a factor of $2.5(\delta \mathrm{D}=9000 \%)$ and seems to 205 homogenize the $\mathrm{D} / \mathrm{H}$ ratio: no significant isotopic anomalous regions can be distinguished 206 within GROpyr over $2170 \mu \mathrm{m}^{2}$ surface area analyzed (Figure 3, figure S3 and Table S2). 
The histograms of the $\delta \mathrm{D}$ values of individual pixels in the IOM of the OCs are 208 different from the histograms usually observed for types 1 and 2 CCs (Figure 4). For Orgueil, 209 the $\delta \mathrm{D}$ histogram is rather narrow, with asymmetry arising from the occurrence of numerous 210 D-rich hotspots on the right side (high values). The tight width of Orgueil IOM histogram 211 indicates that most of the IOM has a homogeneous isotopic ratio representing the bulk value 212 of this IOM, the hot spots only representing some material in the tail of the histogram for the 213 larger values. The contribution of the D-rich hotspot pixels to the total images of Orgueil IOM 214 has previously been shown to be small (Remusat et al., 2009). For the Semarkona and 215 Bishunpur IOM (Figure 4), the $\delta$ D histograms are broader and appear bimodal (see area 2 in 216 both IOM in Figure 4). These histograms are fully consistent with the large differences in the 217 average image $\delta \mathrm{D}$ of Semarkona and Bishunpur IOM (Figure 2) and constitute suitable tools 218 to assess micron-scale isotopic distributions in IOM. Interestingly, the D-poor component in 219 Bishunpur area 2 has the same isotopic signature as the material in Bishunpur area 1. In 220 contrast, the $\delta$ D histogram of the GRO IOM (Figure 4) indicates a homogeneous distribution 221 of $\mathrm{D}$ and the occurrence of a single main D-rich component. 

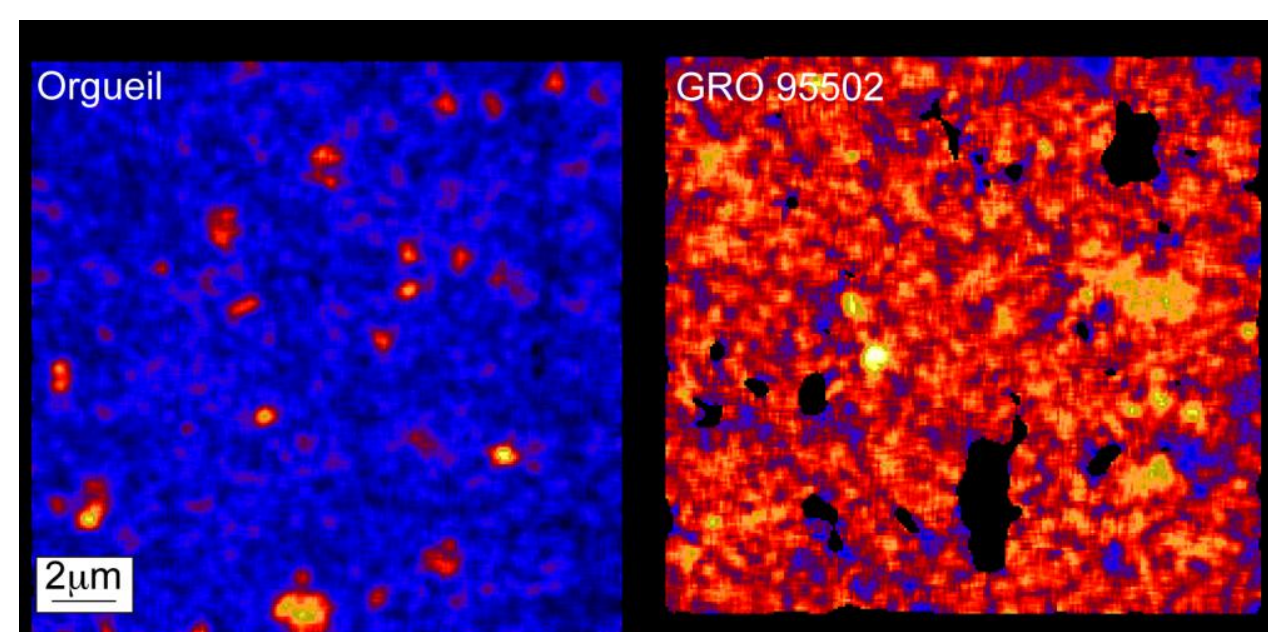

\section{Semarkona Area 1}

\section{Semarkona Area 2}
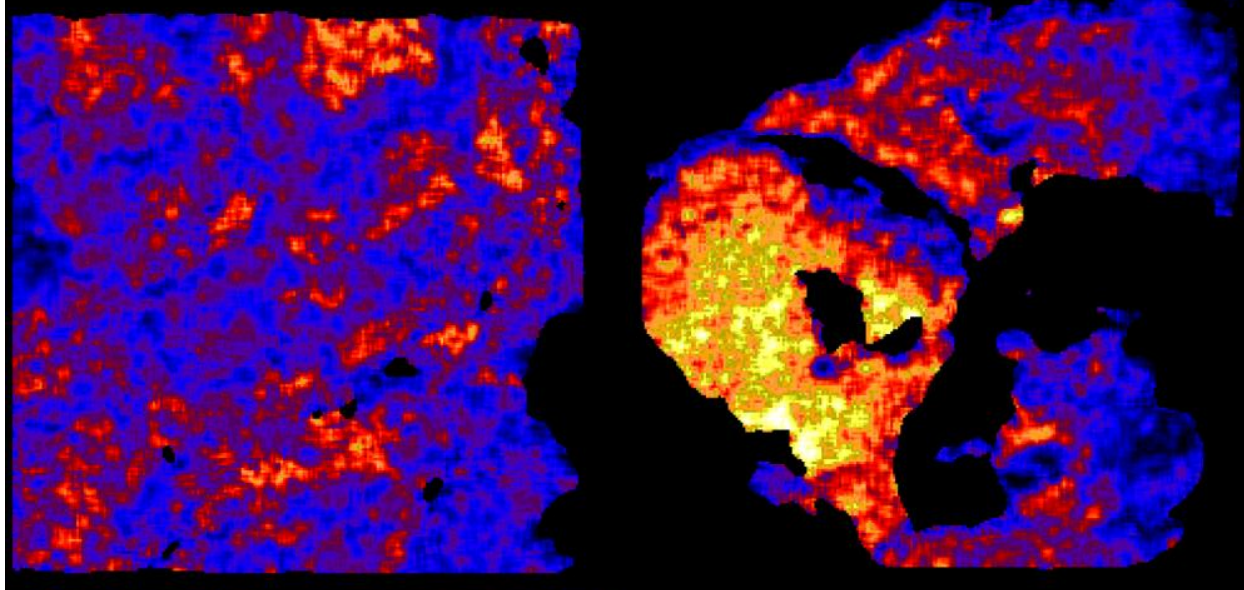

Bishunpur Area 1
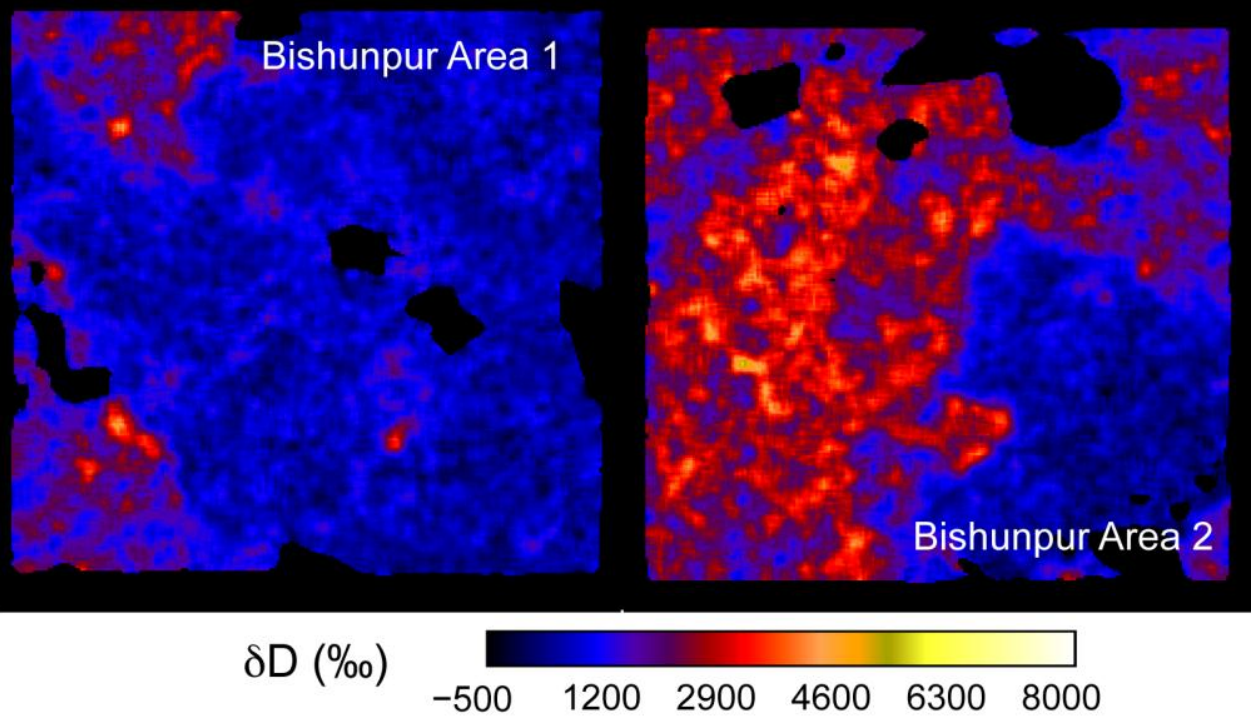

226 Figure 1: NanoSIMS $\delta$ D images of the IOM of Orgueil, GRO 95502, Semarkona and 227 Bishunpur. All the images have the same size; the color scale represents corrected $\delta \mathrm{D}$ values. 228 To illustrate the large-scale variations observed in Semarkona and Bishunpur IOM, two 229 different areas are displayed. 


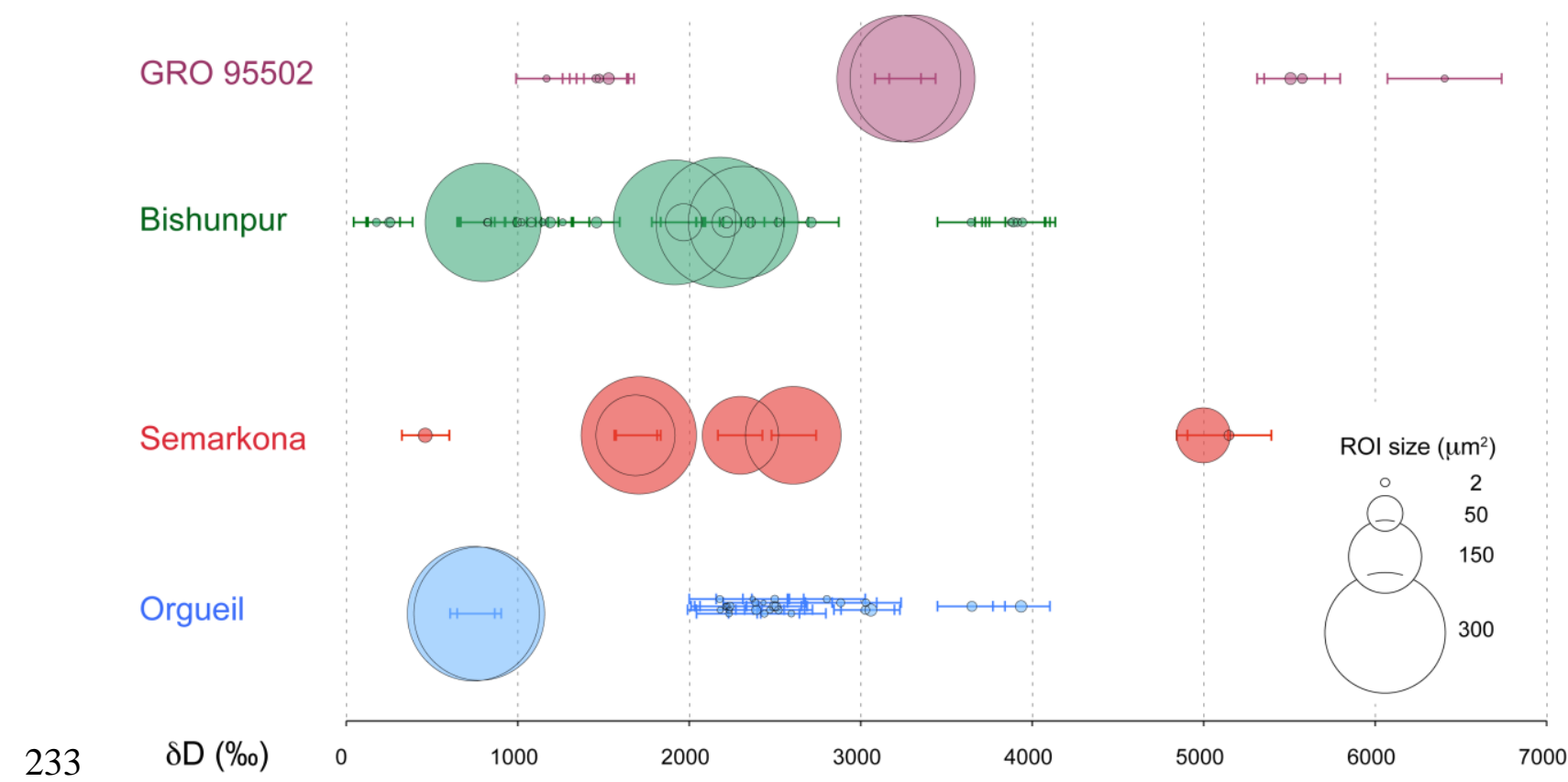

235 Figure 2: $\delta \mathrm{D}$ values determined by NanoSIMS imaging, see also table S2. See text for ROI 236 definition. The size of the disks represents the size of each ROI. The largest circles represent 237 the average image values. In Bishunpur IOM, some regions identified as anomalies in one 238 image may just correspond to "normal" area in other images. 

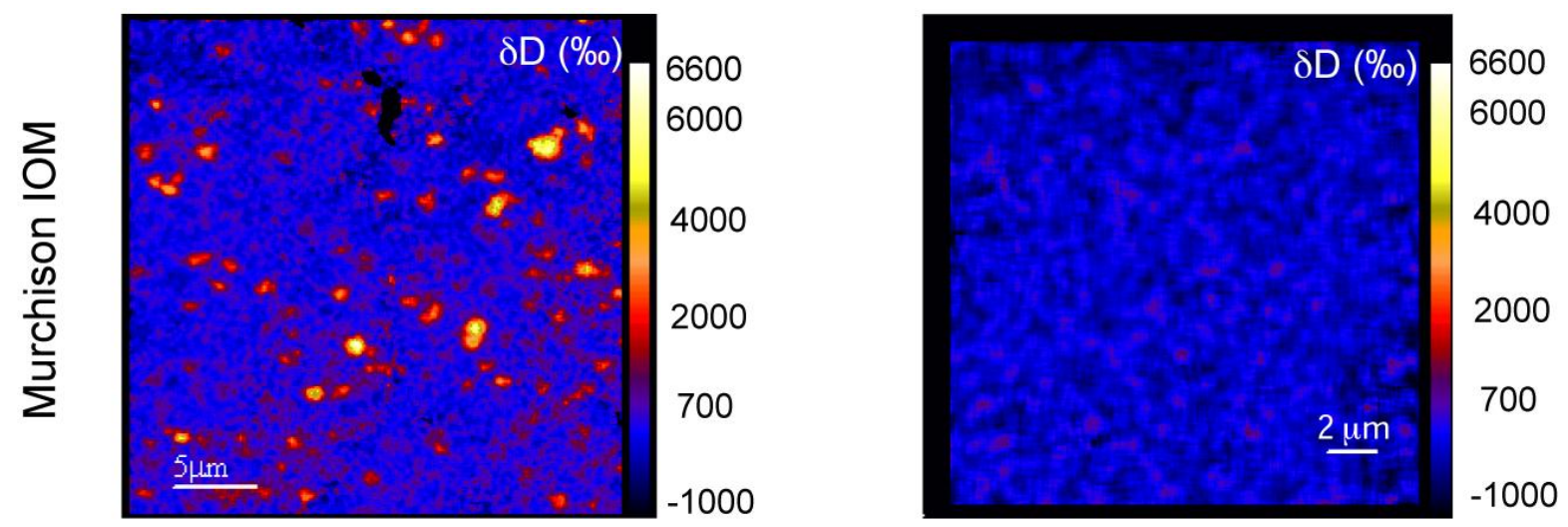

$600^{\circ} \mathrm{C}$ flash heating
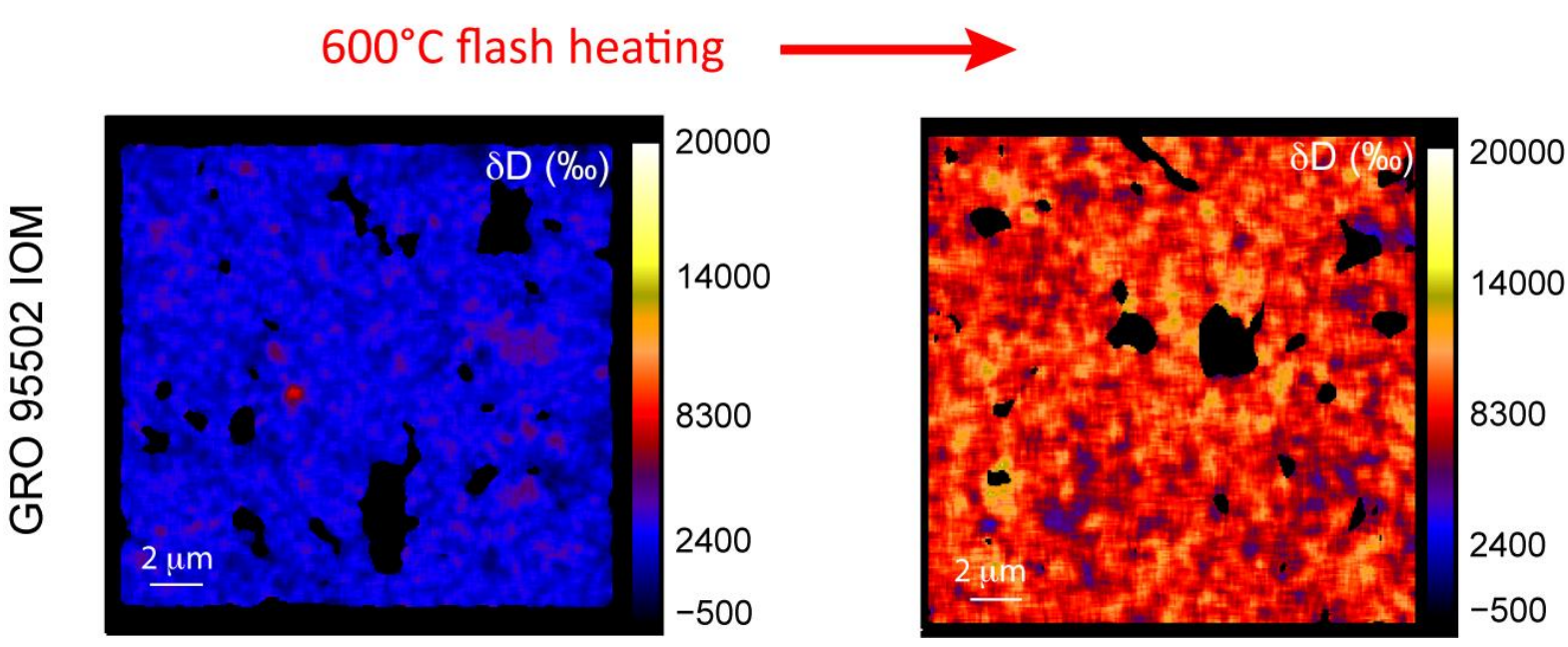

242 Figure 3: Comparative evolution of $\delta$ D NanoSIMS images for Murchison and GRO 95502

$243 \mathrm{IOM}$ upon $600^{\circ} \mathrm{C}$ flash heating. The data for Murchison were taken from Remusat et al.

244 (2009). In order to visualize the isotopic evolution, the color scale is the same before and after

245 heating. The reader is advised that we have reported here, for the unheated GRO IOM on the

246 left hand side, an image of the same areas as in figure 1, but the scale was modified to match

247 the scale of the image of GRO IOM after heating (i.e. GROpyr sample), on the right hand

248 side. For Murchison, the isotopic anomalies are destroyed and some D is lost; it results in a D-

249 depletion. For GRO 95502, we observe an overall D-enrichment. 


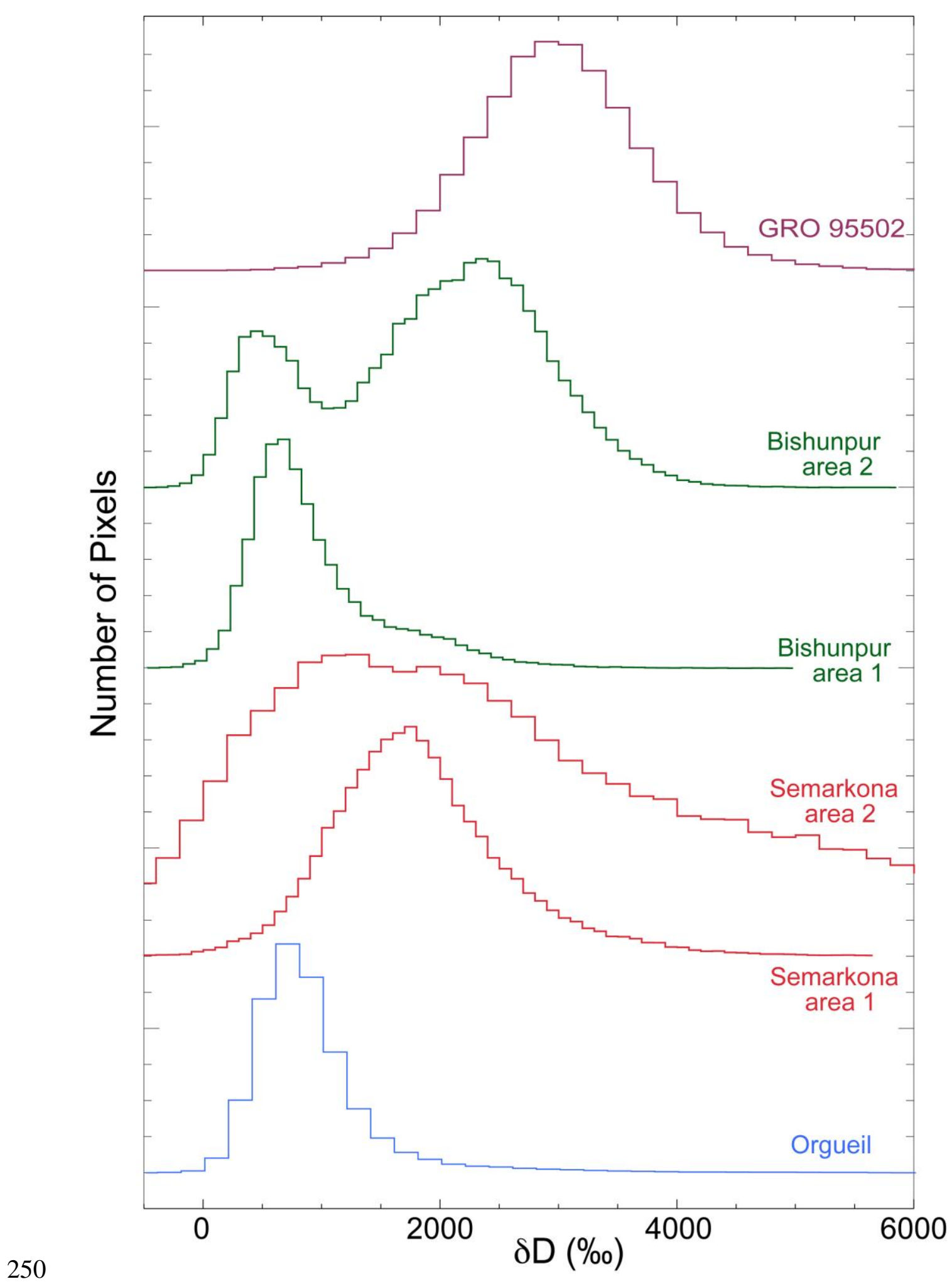

252 Figure 4: pixel (frequency) histograms (pixel value distribution) of the images reported in 253 Figure 1. The vertical axis is in arbitrary units and represents the number of pixels having a 254 given $\delta \mathrm{D}$. $\delta \mathrm{D}$ values are corrected. Bishunpur and Semarkona IOM show clearly distinct 255 histograms. 


\subsection{NanoSIMS imaging of $\mathrm{N}$-isotopes}

257

258 273 increase the measurement uncertainty of each pixel and thus artificially increase the $\delta^{15} \mathrm{~N}$ 274 variability.

Semarkona, Bishunpur and GRO 95502 IOM do not display large-scale $\delta^{15} \mathrm{~N}$ heterogeneities: each investigated IOM exhibits identical mean $\delta^{15} \mathrm{~N}$ values (within error bars) over different regions (Figure 5, Figure 6 and Table S3). The Bishunpur IOM does not exhibit any ${ }^{15} \mathrm{~N}$ anomalies, the Semarkona IOM only one $\left(\delta^{15} \mathrm{~N}>200 \%\right)$ and GRO IOM only two $\left(\delta^{15} \mathrm{~N}>225 \%\right.$ ). The ${ }^{15} \mathrm{~N}$ anomalies of the Semarkona and GRO 95502 IOM are spatially correlated to D anomalies (compare Figures 1 and 5). Some of these ${ }^{15} \mathrm{~N}$ anomalies exhibit significantly lower N/C values than the bulk IOM values. In contrast the Orgueil IOM exhibits numerous micron-scale ${ }^{15} \mathrm{~N}$ positive $\left(120 \%\right.$ < $<\delta^{15} \mathrm{~N}<230 \%$ ) and negative $(-200 \%$ < $\delta^{15} \mathrm{~N}<-70 \%$ ) anomalies; they exhibit the same N/C ratios as the bulk IOM. The occurrence of these anomalies in Orgueil IOM is consistent with data reported for different CCs (Busemann et al., 2006; Nakamura-Messenger et al., 2006; Floss and Stadermann, 2009).

While the Bishunpur IOM shows $\delta^{15} \mathrm{~N}$ histograms similar to that of Orgueil (i.e., a Gaussian curve with a FWHM of 180\%), the Semarkona and GRO 95502 IOM exhibit broader histograms with FWHM of 260\%o (Figure 7). This may result from heterogeneities in $\delta^{15} \mathrm{~N}$ values or, more likely, from a total $\mathrm{N}$ abundance that is three times lower for the IOM of these chondrites compared to Bishunpur and Orgueil IOM. Such a low abundance may indeed 

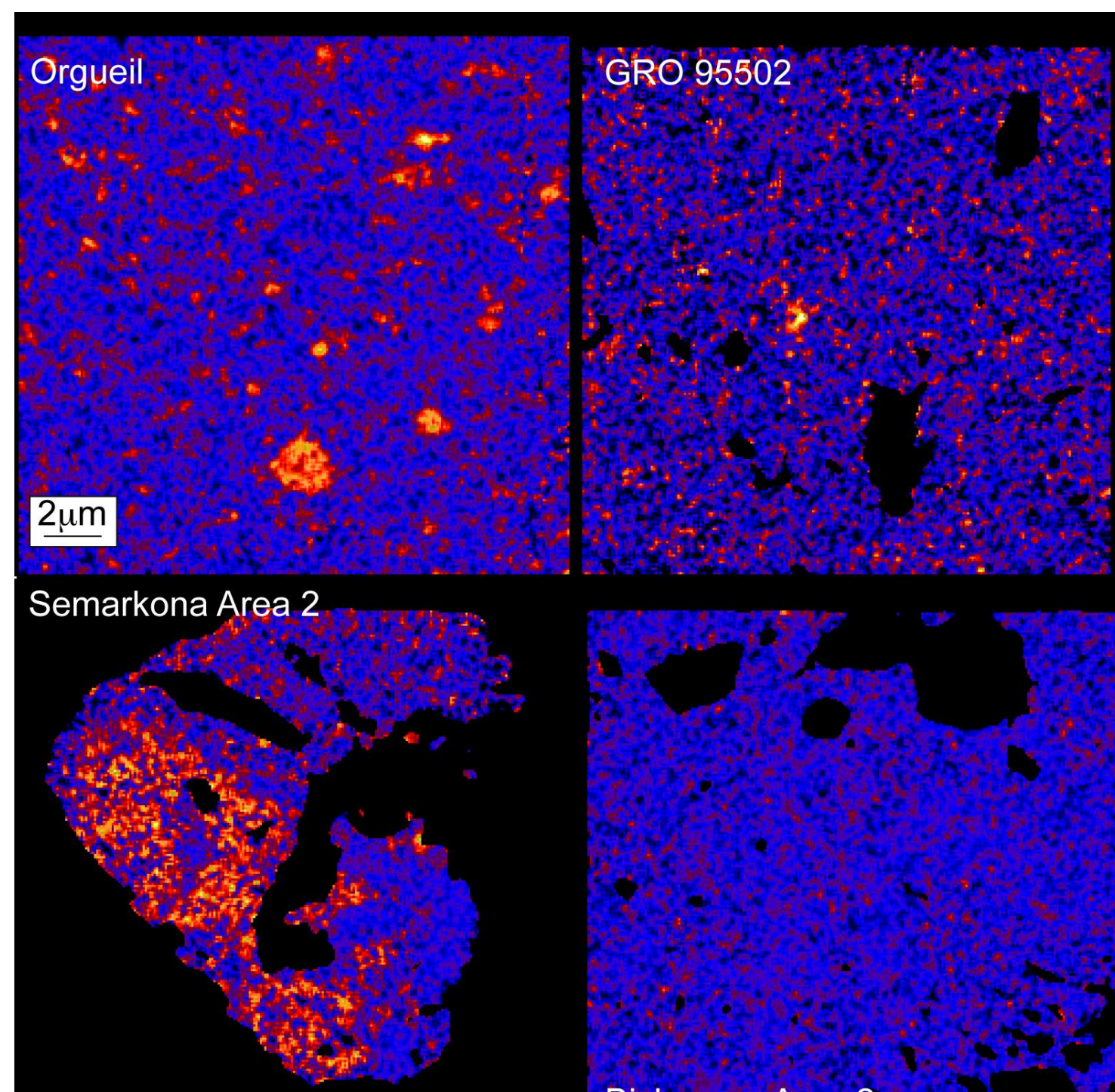

\section{Bishunpur Area 2}

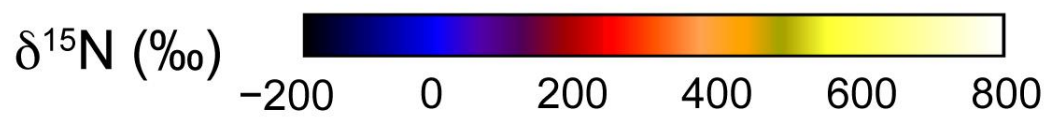

277 Figure 5: NanoSIMS $\delta^{15} \mathrm{~N}$ images of the IOM of Orgueil, GRO 95502, Semarkona and 278 Bishunpur. All the images have the same size; the color scale represents corrected $\delta^{15} \mathrm{~N}$ 279 values. Theses images are located at the same places as the corresponding images in Figure 1. 


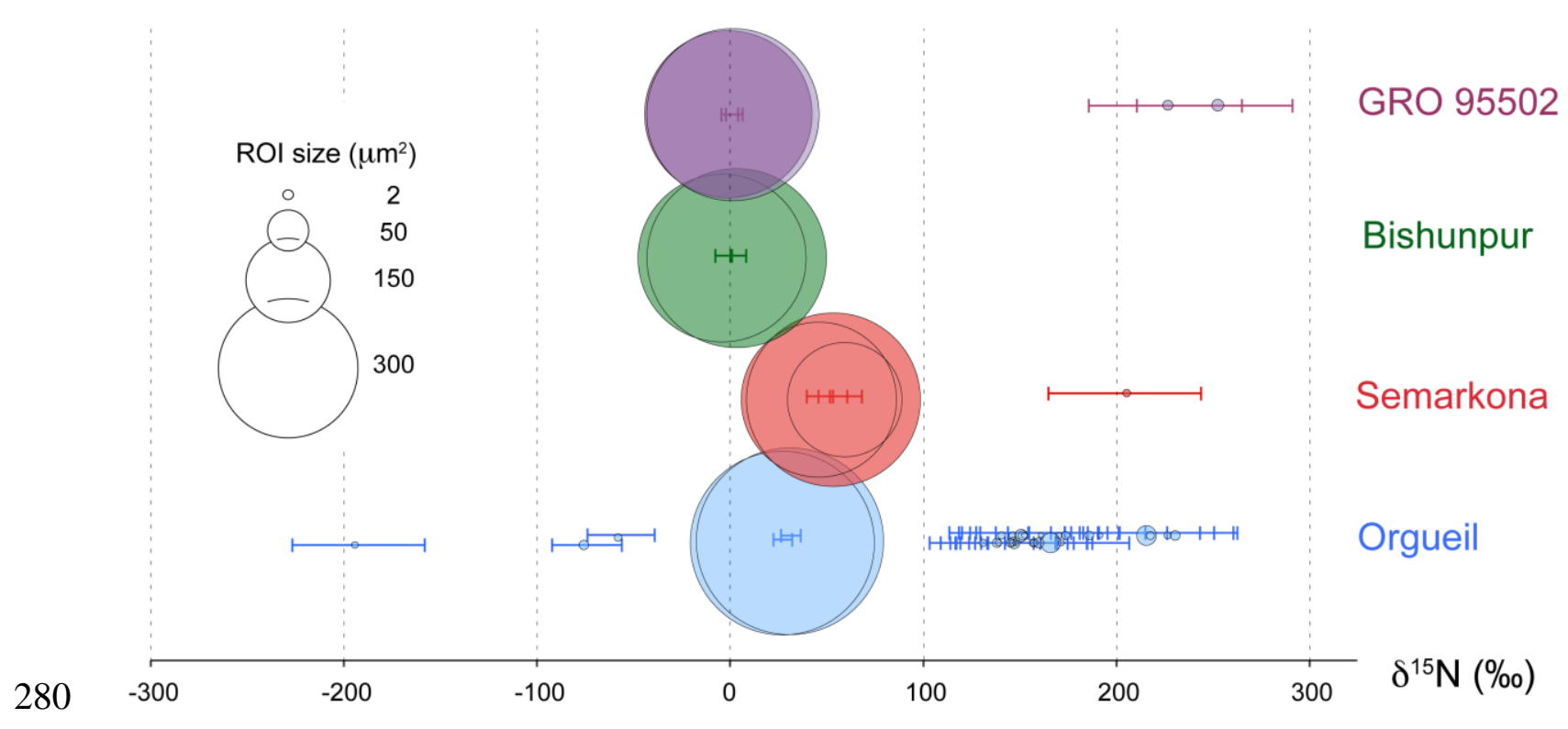

281 Figure 6: $\delta^{15} \mathrm{~N}$ of isotopic anomalies detected in the IOM studied. The size of the disks 282 represents the size of each ROI. The largest circles represent the average image values. 283 Positive anomalies in Semarkona and GRO 95502 are rare and plot in the higher range of 284 Orgueil IOM anomalies. 


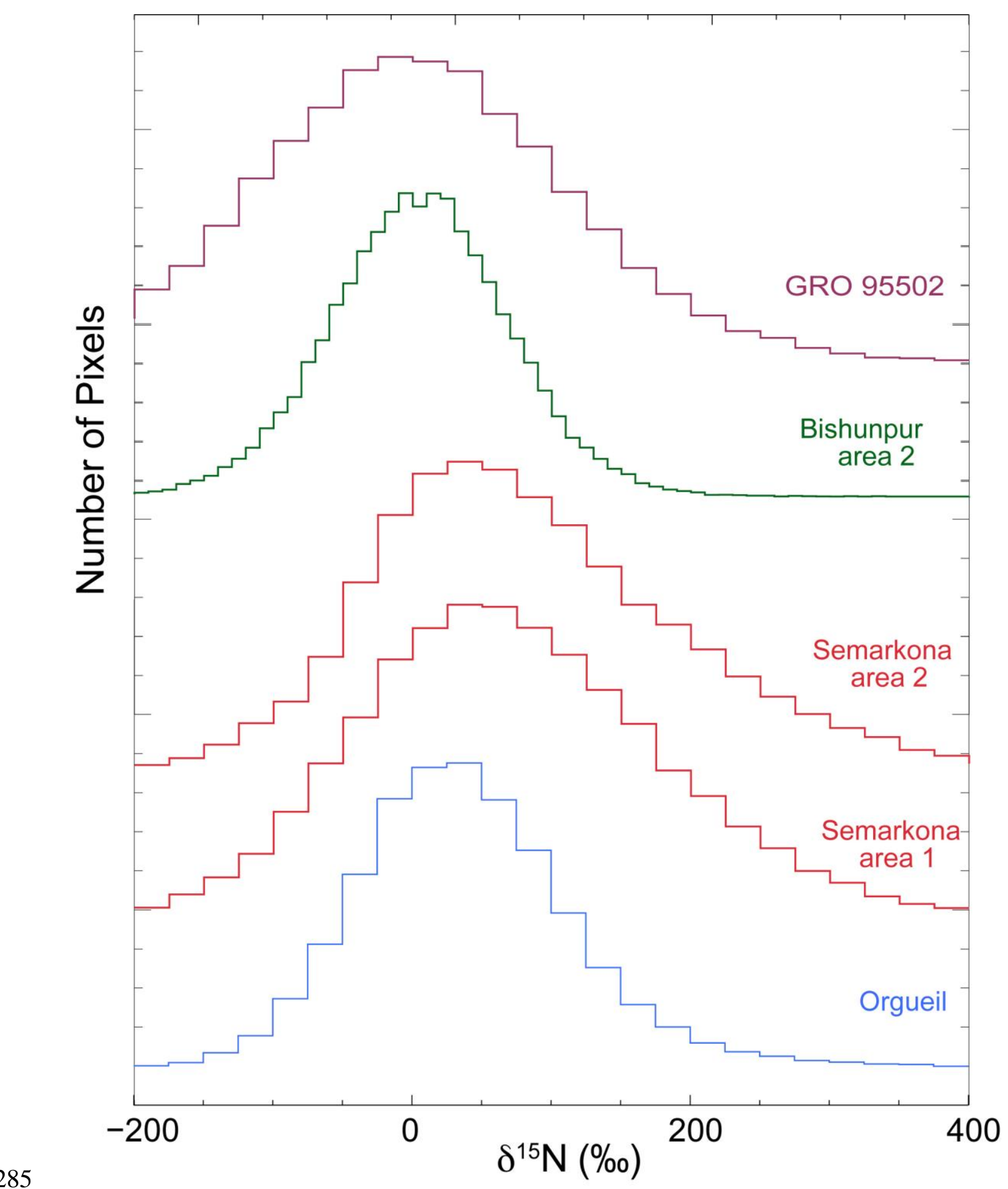

286 Figure 7: pixel (frequency) histograms (pixel value distribution) of the images reported in 287 Figure 5. The vertical axis is in arbitrary units and represents the number of pixels having a 288 certain $\delta^{15} \mathrm{~N}$. Corrected $\delta^{15} \mathrm{~N}$ are reported. 


\subsection{Evolution of the molecular structure of GRO IOM upon heating}

The molecular evolution of OC IOM was investigated by characterizing GRO IOM and GROpyr using both Raman and STXM-based XANES. The Raman signature of the GRO IOM (Figure 8) is consistent with a moderately heated IOM, comparable to Krymka and

\section{4} Inman OM (Quirico et al., 2003), with two main bands of similar intensities: a quite broad D1 band at $1340 \mathrm{~cm}^{-1}$ and a composite $\mathrm{G}+\mathrm{D} 2$ band centered at $1600 \mathrm{~cm}^{-1}$. In contrast to the GRO IOM, GROpyr appears structurally heterogeneous as indicated by the two different Raman spectra that are shown in Figure 8. Of note, all GROpyr Raman spectra exhibit a wellidentified $\mathrm{G}$ band that dominates the signal, as well as D1 and D2 bands that vary in intensity depending on the degree of carbon organization. Overall, these Raman spectra point to thermally evolved organics having experienced partial graphitization (Bernard et al., 2010).

The XANES spectrum of GRO IOM exhibits two prominent absorption peaks at 285.3 and $288.6 \mathrm{eV}$ corresponding to electronic transitions of aromatic and/or olefinic carbon $(\mathrm{C}=\mathrm{C})$ and of carbon in carboxylic functional groups $(\mathrm{COOH})$, respectively. The presence of aliphatic carbon $\left(\mathrm{CH}_{\mathrm{n}}\right)$ can also be seen at $287.5 \mathrm{eV}$. Despite the absence of ketonic carbon (peak at $286.5 \mathrm{eV}$ ), this spectrum appears quite similar to that of CC IOM such as Orgueil or Murchison IOM (De Gregorio et al., 2013; Le Guillou et al., 2014). GROpyr contains significantly more aromatic/olefinic carbons than the GRO IOM together with a lower concentration of aliphatic and carboxylic carbons. The evolution of the XANES signal from GRO IOM to GROpyr is consistent with Raman data and shows molecular evolution during partial graphitization (Bernard et al., 2010).

Taken together, Raman and XANES signatures highlight the similar molecular and structural evolution undergone by both CC IOM (Bonal et al., 2007; Busemann et al., 2007; Cody et al., 2005; Remusat et al., 2008) and OC IOM upon heating (flash pyrolysis at $600^{\circ} \mathrm{C}$ ). 

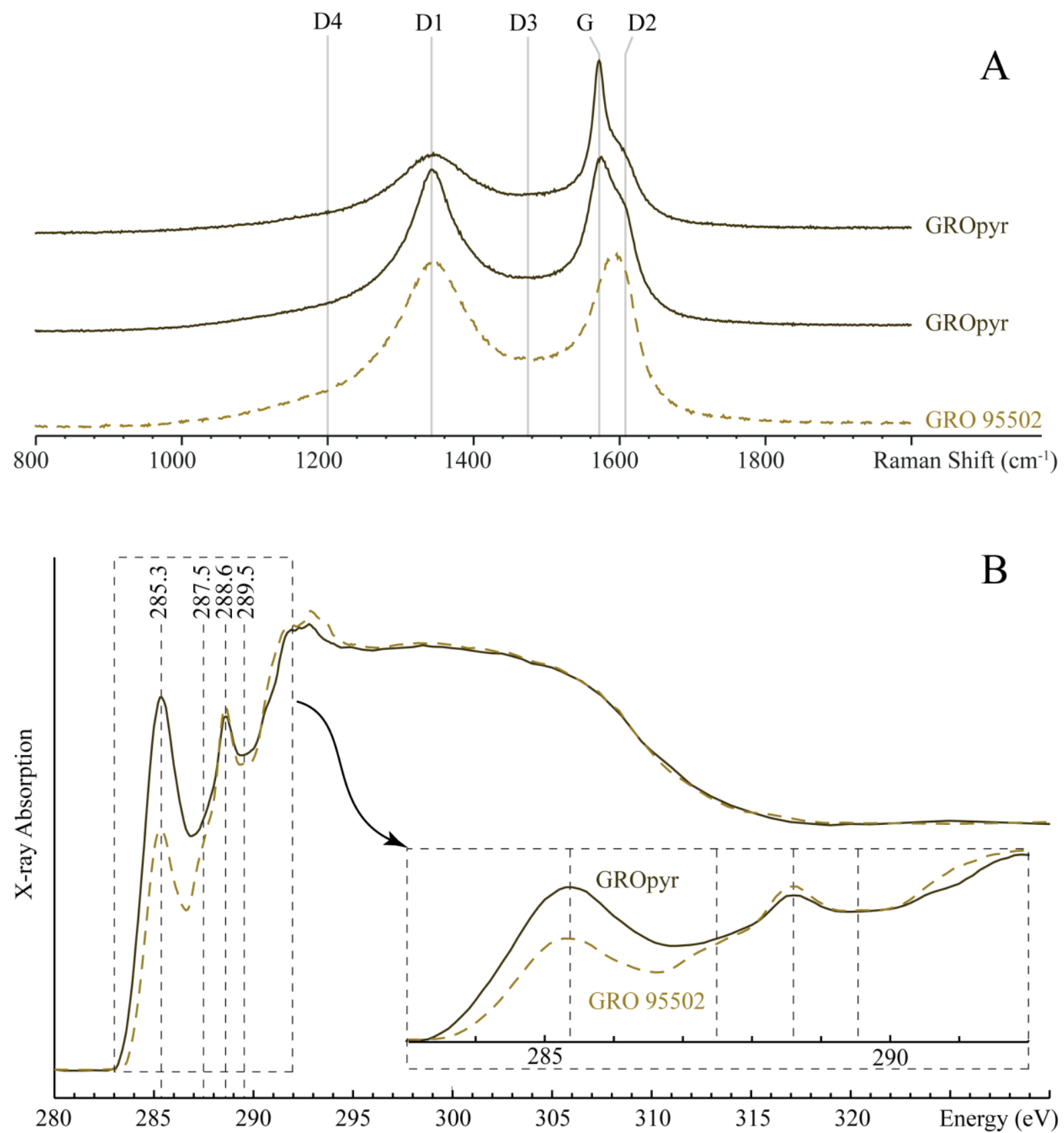

317 Figure 8: Structural and molecular signature evolution of GRO IOM under thermal stress. A:

318 Raman spectra show that GRO IOM is becoming more organized, although it gets 319 heterogeneous. B: XANES spectra of GRO IOM and GROpyr. XANES spectra show that 320 upon heating, GRO IOM is becoming more aromatic and is losing aliphatic C. In addition, $321 \mathrm{COOH}$ groups are less abundant. 


\section{DISCUSSION}

\section{1 $\mathrm{D}$ and ${ }^{15} \mathrm{~N}$ distributions in OC IOM}

The most D-rich organic moieties in the Orgueil IOM are organic radicals (Delpoux et al., 2010), which are heterogeneously distributed in the IOM (Binet et al., 2004). The CI, CM and CR IOM have been described as assemblage of moderately D-rich organic matter associated with organic radicals extremely rich in D that constitute less than $20 \mathrm{vol} \%$ of the total IOM (Remusat et al., 2009). The clusters rich in organic radicals then appear as micronsize D-rich hotspots in NanoSIMS images (Remusat et al., 2009).

In contrast, very few micron-scale D anomalies have been observed in the OC IOM investigated in the present study, consistent with observations reported for the Krymka (LL 3.2) IOM (Alexander et al., 2010). Nevertheless, the D distribution in OC IOM is not homogeneous. Semarkona and Bishunpur IOM seem to be constituted by at least two components that can be resolved at the scale of several microns (Figure 1 and 4). These organic components may have experienced different conditions on the parent body

338 (Alexander et al. 2010) or may have originally been different, i.e. they may have had distinct $\delta \mathrm{D}$ signatures before being accreted. It has been shown that large variations of clay mineral isotope signatures and heterogeneities of organic isotopic signatures are not correlated within

341 the matrix of Semarkona (Piani et al., 2015). This observation strengthens the scenario of the

342 accretion of distinct organic precursors having different isotopic signatures.

The occurrence of large domains with distinct $\mathrm{D} / \mathrm{H}$ is intriguing. Analytical bias during NanoSIMS imaging can be ruled out as such distribution is not observed in terrestrial standards or Orgueil IOM measured under the same experimental conditions. In addition, the average values obtained for Semarkona and Bishunpur IOM are consistent with previous

347 measurements (Alexander et al. 2007), thereby disproving the possibility of an $\mathrm{HF} / \mathrm{HCl}$ 
dissolution artifact (it is unlikely that $\mathrm{HF} / \mathrm{HCl}$ treatments would induce such D-transfer between organic moieties). The occurrence of these large domains with distinct $\mathrm{D} / \mathrm{H}$ may result from the intrinsic nature of the different components of the IOM. OCs exhibit micrometric organic grains together with a diffuse organic component within the matrix, as observed in CCs (Le Guillou et al., 2014; Piani et al., 2015). During HF/HCl dissolution, organic components with similar affinities may gather together forming 10-20 micron large aggregates that may escape destruction during the centrifugation and drying steps.

Despite variable high $\mathrm{D} / \mathrm{H}$ values, the $\mathrm{OC}$ IOM investigated present an almost homogeneous $\mathrm{N}$-isotope composition close to $\delta^{15} \mathrm{~N}=0 \%$. Alexander et al. $(2007 ; 2010)$ reported very large D-enrichment in bulk IOM from various OCs, associated with Earth-like $\mathrm{N}$ isotopic compositions using conventional isotope ratio mass spectrometry. This is in contrast to the CCs where the D-richest IOM also exhibit large ${ }^{15} \mathrm{~N}$-enrichments. Again, such differences may not be explained by different parent body evolution but rather by the accretion of both D- and ${ }^{15} \mathrm{~N}$-rich organics in CRs and a few CMs while only D-rich organics were accreted in OCs. The very few ${ }^{15} \mathrm{~N}$ anomalies in OC IOM exhibit large D-enrichments (making them both $\mathrm{D}$ - and ${ }^{15} \mathrm{~N}$-rich hotspots). Ion-molecule or grain surface chemistry in cold environments could explain both D- and ${ }^{15} \mathrm{~N}$-enrichments, their scarcity possibly resulting from their sensitivity to the thermal metamorphism that occurred on OC parent bodies.

\subsection{Thermal evolution of OC IOM}

While the bulk $\delta^{15} \mathrm{~N}$ signatures do not appear to be sensitive to temperature conditions,

369 the present investigations of three UOCs of increasing metamorphic grades (Semarkona 370 (LL3.0), Bishunpur (LL3.15) and GRO 95502 (L3.2)) demonstrate an increase of $\delta$ D values 371 with increasing metamorphism. In contrast, the D/H and $\mathrm{H} / \mathrm{C}$ ratios of CC IOM decrease with 372 increasing metamorphism (Alexander et al., 2007). In other words, OC and CC IOM exhibit 
opposite trends in the D/H vs. H/C plot (Alexander et al., 2010), even though the structural evolution of OC and CC IOM are similar with increasing temperature (Quirico et al., 2003; Bonal et al., 2007).

Thermal degradation experiments (flash heating) performed on Murchison IOM demonstrated that D-rich hotspots do not sustain temperature (Remusat et al., 2009). Such result makes sense if hotspots concentrate D-rich organic radicals that can be easily thermally destabilized (Remusat et al., 2009). Furthermore, the IOM of the Kainsaz chondrite (CO3.6), which lacks D-rich hotspots, contains a very low amount of radicals (Remusat et al., 2008; Remusat et al., 2009). In contrast, the present study demonstrates that the D/H ratio of GRO IOM increases with increasing temperature (GROpyr D/H is twice as large as GRO IOM) despite a structural and molecular evolution similar to that of CC IOM. Of the two organic components described in 4.1 , one is likely thermally recalcitrant (the D-rich one) while the second evolves into graphitized material through $\mathrm{H}$ loss (its $\mathrm{H} / \mathrm{C}$ decreases with increasing temperature). Indeed, as indicated by Raman and STXM-based XANES spectroscopy, the GRO IOM undergoes a structural reorganization concomitant with an increase of its aromatic/aliphatic carbon ratio as temperature increases. This evolution, typical of the carbonization process (Bernard et al., 2010), is also observed for CC IOM affected by thermal metamorphism (Quirico et al., 2003; Bonal et al., 2007; Busemann et al., 2007, Remusat et al., 2008).

The present contribution thus demonstrates that the organic D-rich reservoirs in OC and CC IOM are different. While CC IOM loses its most D-rich organic reservoir upon heating, OC IOM loses its D-poor organic H reservoir. This is consistent with the evolution from a bimodal D/H histogram, as seen in the Semarkona and Bishunpur IOM, to a more homogeneous distribution in the IOM of the more heated GRO 95502 (Figure 4). This is also 
consistent with NanoSIMS data reported for the IOM of the Krymka 3.2 OC (Alexander et al., 2010).

In summary, in contrast to that of CC IOM, the D-rich component of OC IOM is not borne by organic radicals but rather by thermally recalcitrant organic moieties. This recalcitrance would explain the evolution reported by Alexander et al. $(2007 ; 2010)$ for bulk OC IOM in the $\mathrm{D} / \mathrm{H}$ vs. $\mathrm{H} / \mathrm{C}$ plot, i.e. the increase of the $\mathrm{D} / \mathrm{H}$ ratio of OC IOM may directly result from an increase of metamorphic temperature experienced by OC parent bodies.

404

\subsection{Origin of the D-rich organic reservoir in OCs}

Alexander et al. (2010) suggested that the D-rich component of UOC IOM results from isotopic exchange between IOM and D-rich water, such as the water reported in UOCs by Deloule and Robert (1995). This water would have been enriched in D through distillation process resulting from the progressive reduction of water by reaction with Fe. Yet, Piani et al. (2015) recently reported the absence of spatial correlation between IOM and water D/H ratios as well as huge heterogeneities in the $\delta \mathrm{D}$ values of Semarkona phyllosilicates (up to $\delta \mathrm{D}=$ $10,000 \%$ ) that may not result from distillation processes. Aléon (2010) suggested an alternative hypothesis: he interpreted the D-enrichment of OC IOM as resulting from the presence of presolar organics synthesized in the interstellar medium (ISM). In this model, the $\mathrm{D} / \mathrm{H}$ ratio of OC IOM results from mixing between IOM similar to that of Murchison and extremely D-rich ISM organics ( 1 at.\% at most), with no significant ${ }^{15} \mathrm{~N}$-enrichment relative to solar system organic matter. Hence, according to Aléon (2010) and Piani et al. (2015), both

418 D-rich organics and D-rich water ice from primitive reservoir(s) would have been accreted and preserved within OC parent-bodies. 
fractionation due to ion-molecule reactions (IMR-fl) and mixing between a component akin to

$423 \mathrm{CM}$ chondrites IOM (Murchison) and observed molecules in the interstellar medium (Aléon,

4242010 and references therein). $\mathrm{H}$ and $\mathrm{N}$ isotopic compositions are expressed as isotopic

425 fractionation factors $(\alpha \mathrm{H}$ and $\alpha \mathrm{N})$ between organic matter and the protosolar gas, the

426 composition of which is $\mathrm{D} / \mathrm{H}=21 \times 10^{-6}$ (Geiss and Reeves, 1981) and ${ }^{15} \mathrm{~N} /{ }^{14} \mathrm{~N}=2.36 \times 10^{-3}$

427 (Meibom et al., 2007). The Genesis mission since reported that protosolar ${ }^{15} \mathrm{~N} /{ }^{14} \mathrm{~N}=2.27( \pm$

$4280.03) \times 10^{-3}$ (Marty et al., 2011).

429 The present data are consistent with previous OC IOM data and fall on the mixing 430 curve proposed by Aléon (2010). Most of the D-enrichments reported here are not associated 431 with any significant enrichment in ${ }^{15} \mathrm{~N}$. At the micro-scale, no D anomalies correspond to the 432 ISM organic component with $\mathrm{D} / \mathrm{H}=0.1$ predicted by Aléon (2010). Thus, if mixing has 433 occurred, it has to be at a very fine scale, below a few tens of nanometers, i.e. at the molecular 434 scale. Hence, OC IOM contains interstellar molecular moieties or was formed from ISM 435 precursors that were incorporated into the OC IOM during solar system formation. Of note, D 436 and ${ }^{15} \mathrm{~N}$ anomalies of OC IOM follow the trend defined by the simultaneous $\mathrm{H}$ and $\mathrm{N}$ isotopic 437 fractionation by ion/molecule reactions occurring in the protosolar nebula or in the parent 438 molecular cloud (IMR-fl, Figure 9); hence, they may have formed in the solar system nebula 439 by ion/molecule reactions (Aléon, 2010). 


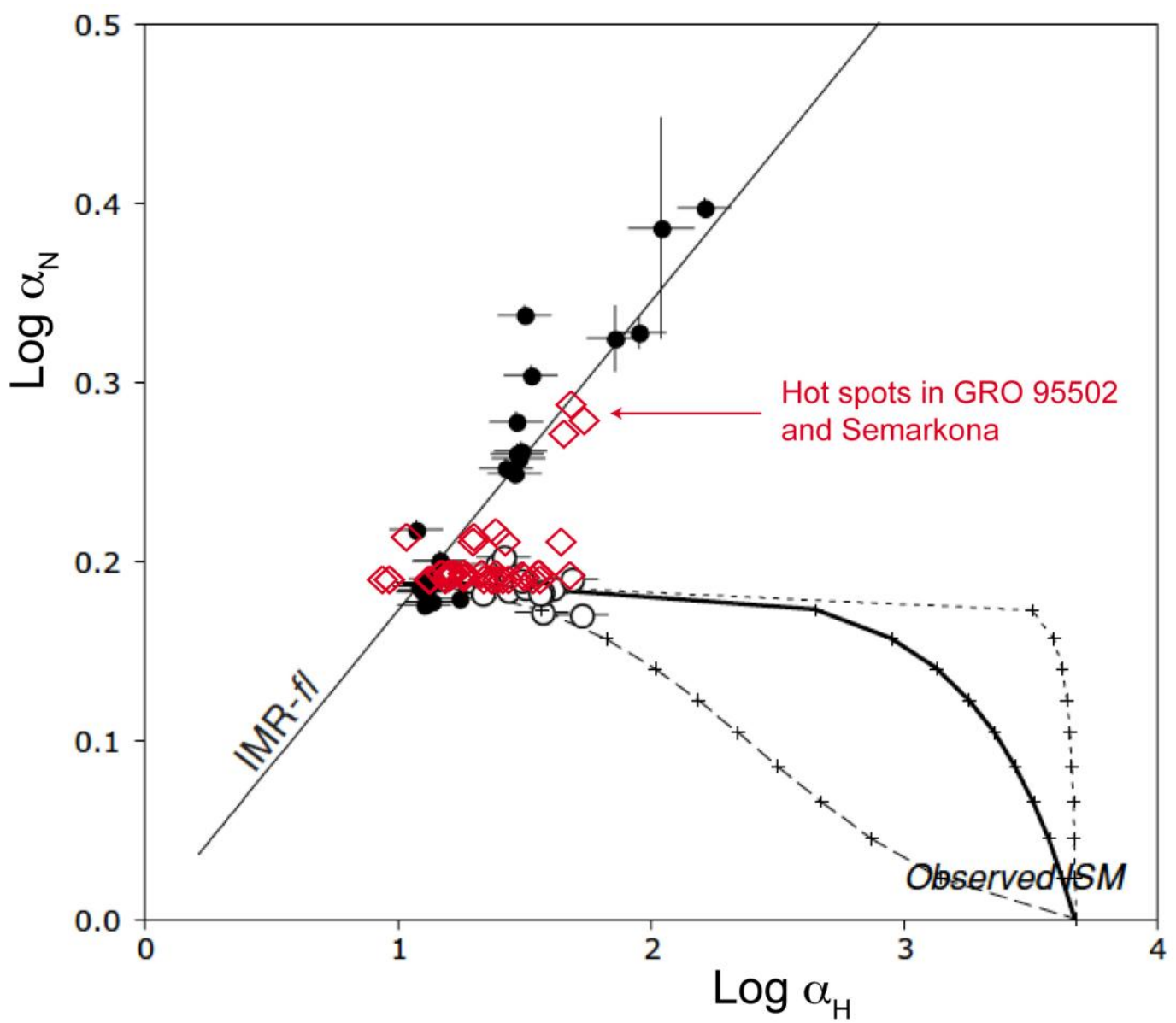

441

444 Figure 9: Comparison of the isotopic composition of OC IOM (this study) with the model by 445 Aléon (2010). The $\mathrm{N}$ isotopic fractionation between organics and the protosolar gas is 446 reported versus the $\mathrm{H}$ isotopic fractionation. Black dots represent sample of group 1 as 447 defined by Aléon: IOM in CC and their hotspots, values taken from the literature until 2010. 448 In open black dots are reported bulk IOM of unequilibrated OC (Alexander et al. 2007). 449 Curves describe mixing between observed interstellar molecules (Observed ISM) and typical $450 \mathrm{CM}$ chondrite IOM with: isochemical mixing (plain curve), mixing with Murchison CHON 451 and ISM HCN (dashed curves) and with Murchison HCN and ISM CHON (doted curved).

452 ISM values are taken from Millar et al. (1989), Turner (2001) and Roueff et al. (2007). 453 Details and references can be found in Aléon (2010). Our data for OC IOM are reported as 454 red open diamonds. It must be noted that three hotspots observed in Semarkona and GRO 45595502 fit with the ion/molecule reaction fractionation line (IMR-fl) defined by (Aléon, 2010). 456 

different. The OC IOM does not exhibit numerous micron-scale isotopic anomalies (both Dand ${ }^{15} \mathrm{~N}$-rich), even in the least metamorphosed, Semarkona. In addition, OC IOM appears to be composed of two main isotopic reservoirs, the D-richer being thermally recalcitrant. Thermal degradation experiments (flash heating) indeed demonstrate that the $\mathrm{D} / \mathrm{H}$ ratio of OC IOM increases with increasing temperature despite a structural and molecular evolution similar to that of CC IOM as indicated by Raman and STXM-based XANES spectroscopy. Based on these observations, we suggest that CCs and OCs did not accrete the same organic precursors, as a consequence of spatial and/or temporal heterogeneities in the solar nebula.

This indicates that the organic content of the protosolar nebula was probably evolving during parent body formation. The common organic precursor concept should be revised, as CC, OC, and enstatite chondrites appear to contain IOM with different properties (Alexander et al., OC IOM may have formed in the ISM, thus supporting the vision of Aléon (2010) stating that 473 OC IOM is "polluted" by minute amount of ISM organics.

\section{ACKNOWLEDGMENTS}

478 having kept the precious IOM of Semarkona and Bishunpur prepared 30 years ago. We thank

479 Aurélien Thomen and François Robert for helpful discussion and Roger Hewins for his comments on the manuscript. The National NanoSIMS Facility at the Museum National 
Ministère délégué à l'Enseignement supérieur et à la Recherche, and the Muséum itself.

483 STXM data were acquired at beamline 5.3.2.2 at the ALS, which is supported by the Director

484 of the Office of Science, Department of Energy, under Contract No. DE-AC02-05CH11231.

485 Special thanks go to David Kilcoyne for his expert support of the 5.3.2.2 STXM at the ALS.

486 L. R. thanks the Programme National de Planétologie (PNP) of CNRS/INSU for financial 487 support. We thank Hikaru Yabuta, Conel Alexander and Editor Bernard Marty for their 488 comments that helped to improve the quality of the paper.

\section{REFERENCES}

Aléon J. (2010) Multiple Origins of Nitrogen Isotopic Anomalies in Meteorites and Comets.

493

494

495

496

497

498

499

500

501

502

503

504

505

506

$$
\text { Astrophys. J. 722, } 1342 .
$$

Alexander C. M. O’D., Barber D. J., and Hutchison R. (1989) The microstructure of Semarkona and Bishunpur. Geochim. Cosmochim. Acta 53, 3045-3057.

Alexander C. M. O'D., Fogel M., Yabuta H., and Cody G. D. (2007) The origin and evolution of chondrites recorded in the elemental and isotopic compositions of their macromolecular organic matter. Geochim. Cosmochim. Acta 71, 4380-4403.

Alexander C. M. O'D., Newsome S. D., Fogel M. L., Nittler L. R., Busemann H., and Cody G. D. (2010) Deuterium enrichments in chondritic macromolecular materialImplications for the origin and evolution of organics, water and asteroids. Geochim. Cosmochim. Acta 74, 4417-4437.

Alleon J., Bernard S., Remusat L., and Robert F. (2015) Estimation of nitrogen-to-carbon ratios of organics and carbon materials at the submicrometer scale. Carbon 84, 290298.

Bernard S., Beyssac O., and Benzerara K. (2008) Raman Mapping Using Advanced LineScanning Systems: Geological Applications. Applied Spectroscopy 62, 1180-1188. 
Bernard S., Beyssac O., Benzerara K., Findling N., Tzvetkov G., and Brown Jr G. E. (2010) XANES, Raman and XRD study of anthracene-based cokes and saccharose-based chars submitted to high-temperature pyrolysis. Carbon 48, 2506-2516.

Beyssac O., Brunet F., Petitet J.-P., Goffé B., and Rouzaud J.-N. (2003) Experimental study of the microtextural and structural transformations of carbonaceous materials under pressure and temperature. European Journal of Mineralogy 15, 937-951.

Binet L., Gourier D., Derenne S., and Robert F. (2002) Heterogeneous distribution of paramagnetic radicals in insoluble organic matter from the Orgueil and Murchison meteorites. Geochim. Cosmochim. Acta 66, 4177-4186.

Binet L., Gourier D., Derenne S., Robert F., and Ciofini I. (2004) Occurence of abundant diradicaloid moieties in the insoluble organic matter from the Orgueil and Murchison meteorites : A fingerprint of its extraterrestrial origin ? Geochim. Cosmochim. Acta 68, 881-891.

Bonal L., Bourot-Denise M., Quirico E., Montagnac G., and Lewin E. (2007) Organic matter and metamorphic history of CO chondrites. Geochim. Cosmochim. Acta 71, 16051623.

Busemann H., Alexander C. M. O'D., and Nittler L. R. (2007) Characterization of insoluble organic matter in primitive meteorites by microRaman spectroscopy. Meteorit. Planet. Sci. 42, 1387-1416.

Busemann H., Young A. F., Alexander C. M. O'D., Hoppe P., Mukhopadhyay S., and Nittler L. R. (2006) Interstellar Chemistry Recorded in Organic Matter from Primitive Meteorites. Science 312, 727-730.

Cody G. D. and Alexander C. M. O’D. (2005) NMR studies of chemical and structural variation of insoluble organic matter from different carbonaceous chondrite groups. Geochim. Cosmochim. Acta 69, 1085-1097. 
De Gregorio B. T., Stroud R. M., Nittler L. R., Alexander C. M. O'D., et al. (2013) Isotopic and chemical variation of organic nanoglobules in primitive meteorites. Meteorit. Planet. Sci. 48, 904-928.

Delpoux O., Gourier D., Vezin H., Binet L., Derenne S., and Robert F. (2010) Biradical character of D-rich carriers in the insoluble organic matter of carbonaceous chondrites: A relic of the protoplanetary disk chemistry. Geochim. Cosmochim. Acta 75, 326-336.

Floss C. and Stadermann F. J. (2009) High Abundances of Circumstellar and Interstellar CAnomalous Phases in the Primitive CR3 Chondrites QUE 99177 and MET 00426. Astrophys. J. 697, 1242-1255.

Geiss J. and Reeves H. (1981) Deuterium in the solar system. Astron. Astrophys. 93, 189-199.

Hayatsu R., Winans R. E., Scott R. G., McBeth R. L., Moore L. P., and Studier M. H. (1980) Phenolic ethers in the organic polymer of the Murchison meteorite. Science 207, 12021204

Hitchcock A. P., Dynes J. J., Johansson G., Wang J., and Botton G. (2008) Comparison of NEXAFS microscopy and TEM-EELS for studies of soft matter. Micron 39, 311-319.

Kilcoyne A. L. D., Tyliszczak T., Steele W. F., Fakra S., et al. (2003) Interferometercontrolled scanning transmission X-ray microscopes at the Advanced Light Source. Journal of Synchrotron Radiation 10, 125-136.

Le Guillou C., Bernard S., Brearley A. J., and Remusat L. (2014) Evolution of organic matter in Orgueil, Murchison and Renazzo during parent body aqueous alteration: In situ investigations. Geochim. Cosmochim. Acta 131, 368-392.

Marty B., Chaussidon M., Wiens R. C., Jurewicz A. J. G., and Burnett D. S. (2011) A ${ }^{15}$ Npoor isotopic composition for the Solar System as shown by Genesis Solar Wind samples. Science 332, 1533-1536. 
Meibom A., Krot A. N., Robert F., Mostefaoui S., Russell S. S., Petaev M. I., and Gounelle M. (2007) Nitrogen and Carbon Isotopic Composition of the Sun Inferred from a High-Temperature Solar Nebular Condensate. Astrophys. J. 656, L33-L36.

Millar T. J., Bennett A., and Herbst E. (1989) Deuterium fractionation in dense interstellar clouds. Astrophys. J., 340, 906-920.

Nakamura-Messenger K., Messenger S., Keller L. P., Clemett S. J., and Zolensky M. E. (2006) Organic gobules in the Tagish Lake meteorite: remnants of the protosolar disk. Science 314, 1439-1442.

Orthous-Daunay F. R., Quirico E., Lemelle L., Beck P., deAndrade V., Simionovici A., and Derenne S. (2010) Speciation of sulfur in the insoluble organic matter from carbonaceous chondrites by XANES spectroscopy. Earth Planet. Sci. Let. 300, 321328.

Piani L., Remusat L., and Robert F. (2012a) Determination of the H Isotopic Composition of Individual Components in Fine-Scale Mixtures of Organic Matter and Phyllosilicates with the Nanoscale Secondary Ion Mass Spectrometry. Anal. Chem. 84, 10199-10206.

Piani, L., Robert, F., Beyssac, O., Binet, L., Bourot-Denise, M., Derenne, S., Le Guillou, C., Marrocchi, Y., Mostefaoui, S., Rouzaud, J.-N., Thomen, A. (2012b). Structure, composition, and location of organic matter in the enstatite chondrite Sahara 97096 (EH3). Meteorit. Planet. Sci. 47, 8-29.

Piani L., Robert F., and Remusat L. (2015) Micron-scale D/H heterogeneity in chondrite matrices: A signature of the pristine solar system water? Earth Planet. Sci. Let. 415, $154-164$.

Quirico E., Raynal P. I., and Bourot-Denise M. (2003) Metamorphic grade of organic matter in six unequilibrated ordinary chondrites. Meteorit. Planet. Sci. 38, 795-811. 
Ravel B. and Newville M. (2005) ATHENA, ARTEMIS, HEPHAESTUS: data analysis for X-ray absorption spectroscopy using IFEFFIT. Journal of Synchrotron Radiation 12, $537-541$.

Remusat L., Derenne S., Robert F., and Knicker H. (2005) New pyrolytic and spectroscopic data on Orgueil and Murchison Insoluble Organic Matter : A different origin than soluble ? Geochim. Cosmochim. Acta 69, 3919-3932.

Remusat L., Le Guillou C., Rouzaud J.-N., Binet L., Robert F., and Derenne S. (2008) Molecular study of the Insoluble Organic Matter of Kainsaz CO3 carbonaceous chondrite: comparison with CI and CM IOM. Meteorit. Planet. Sci. 43, 1099-1111.

Remusat L., Robert F., Meibom A., Mostefaoui S., et al. (2009) Proto-Planetary Disk Chemistry Recorded by D-Rich Organic Radicals in Carbonaceous Chondrites. Astrophys. J. 698, 2087-2092.

Remusat L (2015) Organics in primitive meteorites In EMU Notes in Mineralogy - volume 15: Planetary Mineralogy (eds. M.R. Lee and H. Leroux). The European Mineralogical Union. ISBN: 978-0903056-55-7. pp. 33-65 (Chapter 2).

Robert F. and Epstein S. (1982) The concentration and isotopic composition of hydrogen, carbon and nitrogen in carbonaceous meteorites. Geochim. Cosmochim. Acta 46, 8195.

Roueff E., Herbst E., Lis D.C. and Phillips T.G. (2007) The Effect of an Increased Elemental D/H Ratio on Deuterium Fractionation in the Cold Interstellar Medium. Astrophys. J. 661, L159-L162.

Sears D. W. G., Morse A. D., Hutchison R., Guimon R. K., et al. (1995) Metamorphism and aqueous alteration in low petrographic type ordinary chondrites. Meteoritics 30, 169. 
604 Solomon D., Lehmann J., Kinyangi J., Liang B., et al. (2009) Carbon (1s) NEXAFS

605

606

607

608

609

610

611

612

613

614

615

616

617

618

619

620

621 Spectroscopy of Biogeochemically Relevant Reference Organic Compounds. Soil Sci. Soc. Am. J. 73, 1817-1830.

Thomen A., Robert F., and Remusat L. (2014) Determination of the nitrogen abundance in organic materials by NanoSIMS quantitative imaging. Journal of Analytical Atomic Spectrometry 29, 512-519.

Turner B. E. (2001) Deuterated molecules in translucent and dark clouds. Astrophys. J. Sup, 136, 579-629.

Wang J., Morin C., Li L., Hitchcock A. P., Scholl A., and Doran A. (2009) Radiation damage in soft X-ray microscopy. Journal of Electron Spectroscopy and Related Phenomena 170, 25-36.

Weisberg M. K., McCoy T. J., and Krot A. N. (2006) Systematics and Evaluation of Meteorite Classification. In Meteorites and the Early Solar System II (eds. D. Lauretta and H. Y. J. J. McSween). University of Arizona Press, Tucson, USA. pp. 19-52.

Yang J. and Epstein S. (1983) Interstellar organic matter in meteorites. Geochim. Cosmochim. Acta 47, 2199-2216.

Zanda B., Bourot-Denise M., and Hewins R. H. (1995) Condensate sulfide and its metamorphic transformations in primitive chondrites. Meteoritics 30, 605. 